\title{
Numerical simulations of single conjugate adaptive optics systems
}

\section{Matthew C. Britton}

Matthew C. Britton, "Numerical simulations of single conjugate adaptive optics systems," Proc. SPIE 5490, Advancements in Adaptive Optics, (25 October 2004); doi: 10.1117/12.552325

Event: SPIE Astronomical Telescopes + Instrumentation, 2004, Glasgow, United Kingdom 


\title{
Numerical simulations of single conjugate adaptive optics systems
}

\author{
Matthew C. Britton \\ California Institute of Technology, Pasadena, CA 91125 USA
}

\begin{abstract}
This paper presents a series of studies of single conjugate adaptive optics systems that use numerical simulation to investigate aspects of system performance not addressed by traditional statistical metrics. These studies include investigations of temporal control loop dynamics and quantitative comparisons of system performance using different types of reconstructors.
\end{abstract}

Keywords: Adaptive optics, telescopes, diffraction, simulation, turbulence

\section{INTRODUCTION}

Single conjugate adaptive optics systems are traditionally analyzed using statistical measures of performance. ${ }^{1}$ These performance estimates rely on the independent estimation of terms such as fitting error, measurement error and servo error. The RSS of these terms then yields an overall estimate of the residual wavefront error. This methodology is very effective for many purposes, but has little predictive value in capturing nonlinear effects or in describing the detailed temporal behavior of the adaptive optics system. These details become important in a number of different circumstances. Examples include quantitative predictions on the relative performance of different reconstruction algorithms, in tolerancing the system design, and in the analysis of the dynamical stability of the control loop. For these purposes, detailed time domain numerical simulations can serve an important role in the analysis of adaptive optics systems.

This paper presents a series of numerical simulations that aim to investigate aspects of the adaptive optics system not addressed by traditional statistical performance metrics. The simulations presented here were performed using Arroyo' : a software library that supports time domain simulations of wave propagation through turbulence and adaptive optics systems. These simulations modeled a single conjugate adaptive optics system guiding on a natural guide star with a Shack Hartmann wavefront sensor. Wave propagation through the lenslet array was included in the simulation, but detection of the wavefront sensor signal was assumed noiseless. Adaptive correction was performed using a tip tilt mirror and a deformable mirror with a rectilinear actuator grid. A pyramidal influence function was assumed for the deformable mirror actuators. Reconstruction was effected using least squares reconstructors, which were generated assuming pyramidal actuator influence functions. Integral control was employed in driving both the tip tilt and deformable mirror control loops. Unless otherwise specified in the text, the parameters used in the simulations are listed in Table 1.

\section{FITTING ERROR}

The first study presented in this paper aimed to validate the results of numerical simulation against the statistical estimates of fitting error. In the absence of dynamical evolution of the atmospheric turbulence, on-axis predictions of $\mathrm{AO}$ system performance consist of two terms: fitting error and centroid measurement error. ${ }^{1}$ The fitting error term takes the form

$$
\sigma_{\phi}^{2}=\alpha_{F}\left(\frac{d}{r_{0}}\right)^{\frac{5}{3}}
$$

where $d$ is the subaperture size, $r_{0}$ is the Fried parameter, and $\alpha_{F}$ is a factor related to the geometrical form of the deformable mirror. For continuous facesheet mirrors, $\alpha_{F}=.28$. The centroid measurement error term nominally vanishes in the absence of noise in the wavefront sensor camera. In contrast, numerical simulation

Further author information: Send correspondence to Matthew Britton: E-mail: mbritton@astro.caltech.edu 


\begin{tabular}{ll} 
Parameter & Value \\
\hline \hline Wavefront pixel scale & $2 \mathrm{~cm}$ \\
Atmospheric phase screen pixel scale & $2 \mathrm{~cm}$ \\
Subharmonic method & Lane $^{3}-3$ levels \\
Turbulence profile & Six layer Cerro Pachon ${ }^{4}$ \\
Fried parameter & $.15 \mathrm{~cm}$ at $.5 \mu \mathrm{m}$ \\
Turbulence power spectrum & Komolgorov \\
Sensing wavelength & $.6 \mu \mathrm{m}-$ monochromatic \\
Science wavelength & $2.2 \mu \mathrm{m}-$ monochromatic \\
Oversampling of science PSF & 8 times Nyquist \\
Atmospheric propagation & Geometric \\
Actuator to lenslet registration & Fried \\
Aperture geometry & Circular \\
Pixels per lenslet & 32 \\
Limiting tip tilt mirror velocity & $100 \mathrm{rad} / \mathrm{sec}$ \\
Limiting deformable mirror actuator velocity & $2.67 \mathrm{e}-4 \mathrm{~meters} / \mathrm{sec}$ \\
\hline
\end{tabular}

Table 1. Parameters used for the numerical simulations described in this paper, unless otherwise specified in the text.

of an adaptive optics system captures additional effects. The modeling of wave propagation through the lenslet array yields spots that are corrupted by turbulence induced wavefront phase errors. These corrupted spots act as a source of noise when computing centroids from the wavefront sensor data. The simulation also captures the detailed aspects of reconstruction and control. In this section, a comparison of the statistical prediction is made to the results of numerical simulations.

In this study, a 5 meter circular aperture was adopted, and the number of subapertures across the pupil varied between 10 and 30 . The wind velocity of each layer in the atmospheric turbulence model was set to zero to isolate the effects of fitting error. In the simulation, the tip tilt control loop was closed and this mirror was allowed to stabilize. Then the deformable mirror control loop was closed, and the simulation was terminated well after the deformable mirror stablilized. The science point spread function (PSF) was used to compute the Strehl ratio by direct comparison of its peak value to that of a PSF uncorrupted by atmospheric turbulence. The residual wavefront phase error was then computed from the Strehl ratio using the Marechal approximation. Statistical variations in the simulated residual wavefront errors arise due to variations in the random realizations of atmospheric turbulence. To provide a statistically valid comparison, twenty to forty independent simulations were performed for each adaptive optics system configuration.

The residual wavefront phase errors from these simulations are shown in Figure 1, and are in agreement with the fitting error estimates. This indicates that the additional effects captured in simulation do not appreciably imapct the performance of the adaptive optics (AO) system for the parameters listed in Table 1. The residuals show scatter that decreases with the number of subapertures in the AO system. This is a well understood effect, and arises from the fact that the characteristic spatial coherence scale of the residual phase errors decreases with increasing order of the $\mathrm{AO}$ system. Consequently, one is averaging over an increasing number of independent zones in the pupil plane, yielding a smaller variance for the residual wavefront phase errors.

\section{THIRTY METER SEGMENTED MIRROR APERTURES}

There are a number of proposals for constructing telescopes with apertures from twenty to one hundred meters in diameter. These projects will rely heavily on adaptive optics systems to overcome the effects of atmospheric turbulence. Numerical simulations can play an important role in identifying potential problems unique to large actuator count adaptive optics systems on large aperture telescopes. This section presents simulations of a 64x64 subaperture adaptive optics system compensating a 30 meter segmented mirror telescope. The primary difference in the requirements for an adaptive optics system on such a large aperture telescope is the increase in the wavefront phase errors that must be compensated. The piston and tilt removed phase variance scales with 

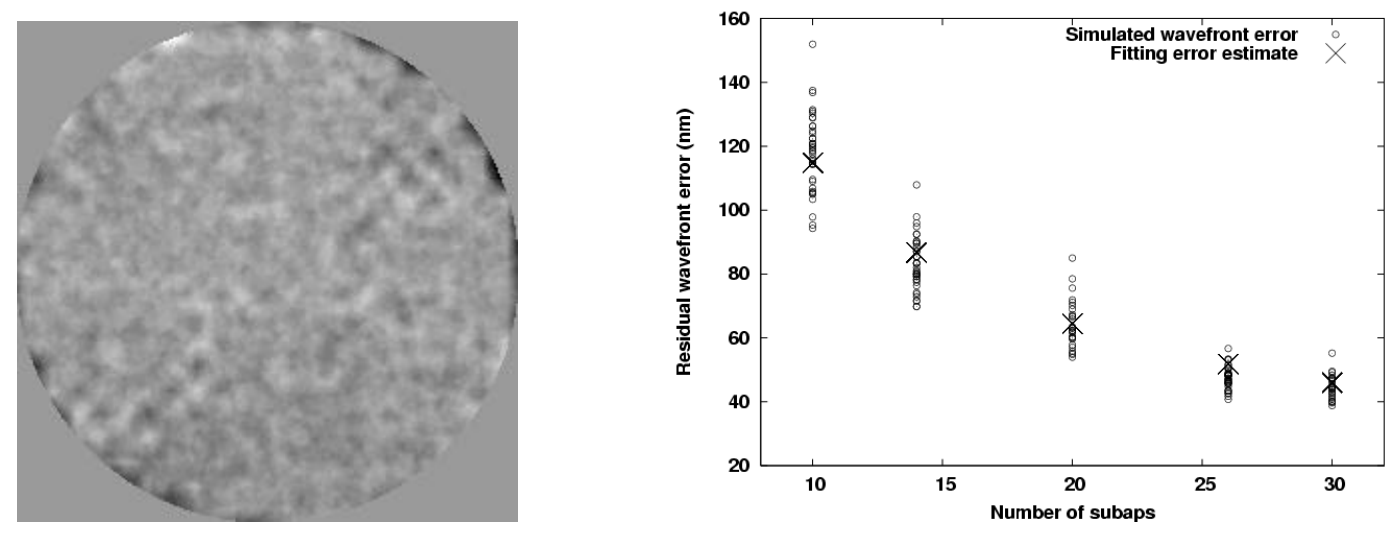

Figure 1. Residual wavefront phase errors after compensation by an adaptive optics system. On the left is shown residual wavefront phase errors in the pupil plane of a 5 meter telescope after compensation by an AO system with 20 subapertures across the pupil. On the right is shown the residual wavefront errors as a function of the order of the AO system.

aperture diameter $D$ as $\sigma^{2}=.134\left(D / r_{0}\right)^{5 / 3}$. For the Fried parameter in Table 1, the rms phase error amounts to $.54 \mu \mathrm{m}$ for a 5 meter aperture, and $2.4 \mu \mathrm{m}$ for a 30 meter aperture.

The simulations were performed on a 30 meter segmented aperture primary. The edge length of each segment was taken to be .5 meters, and the gap size between segments was chosen as 4 millimeters. The simulation was performed with a Fried parameter of .25 meters at $.5 \mu \mathrm{m}$. With these two exceptions, the parameters of the simulation are those in Table 1. As in the previous section, the wind velocity of each of the layers in the atmospheric model was set to zero. The simulation was executed on 1 millisecond timesteps, and the control loops were updated at this rate.

The feature most notably different from simulations of lower actuator count adaptive optics systems on smaller aperture telescopes was in closing the deformable mirror control loop. Here only a qualitative description of this behavior is presented. Due to the large number of actuators in the adaptive optics system, different regions of the deformable mirror equilibrated rapidly to different mean values. This produced a number of distinct patches in the compensated wavefront phase entering the wavefront sensor. An illustration of this behavior is shown in Figure 2. At the boundary of these patches, the wavefront phase tended towards a discontinuity, so that the magnitudes of the phase slopes grew to values much larger than those expected for a Komolgorov turbulence spectrum. These slopes were large enough to seriously distort the point spread functions of lenslets lying on the boundary, yielding poor estimates of the centroids in these subapertures. This then led to the reconstructor yielding poor estimates of the wavefront phase, which significantly slowed the convergence of the control loop. This particular simulation required about 40 cycles of the control loop to equilibrate the deformable mirror surface, whereas the simulations presented in the previous section required only a few cycles.

It should be noted that the simulation modeled the temporal response of the actuators in the deformable mirror as having a limiting velocity. However, the surface of the deformable mirror was never limited in its motion by this velocity. It is also important to note that the simulation did not enforce any interactuator stroke limitation, and relative displacements of up to a few microns occurred between adjacent actuators on the deformable mirror. If this exceeds the interactuator stroke limitation on the deformable mirror, then enforcing the actual limitation may mitigate this behavior to some degree. However, it seems likely that this sort of behavior is not an artifact of numerical simulation, but represents a real and undesirable effect. Designs for AO systems on large aperture telescopes should aim to avoid this type of behavior. One possible technique would be to close the loop on the first several Zernike modes to reduce the overall wavefront phase error, and then switch to a zonal basis to remove the remaining aberrations. Another possibility would be to enforce an interactuator stroke limitation on the deformable mirror commands even if this mirror does not actually have this limitation. Such a limitation would ensure that the phase slopes always remain within a range that maintains the integrity of the lenslet spots. Possible implementations of these techniques are left for future investigation. 

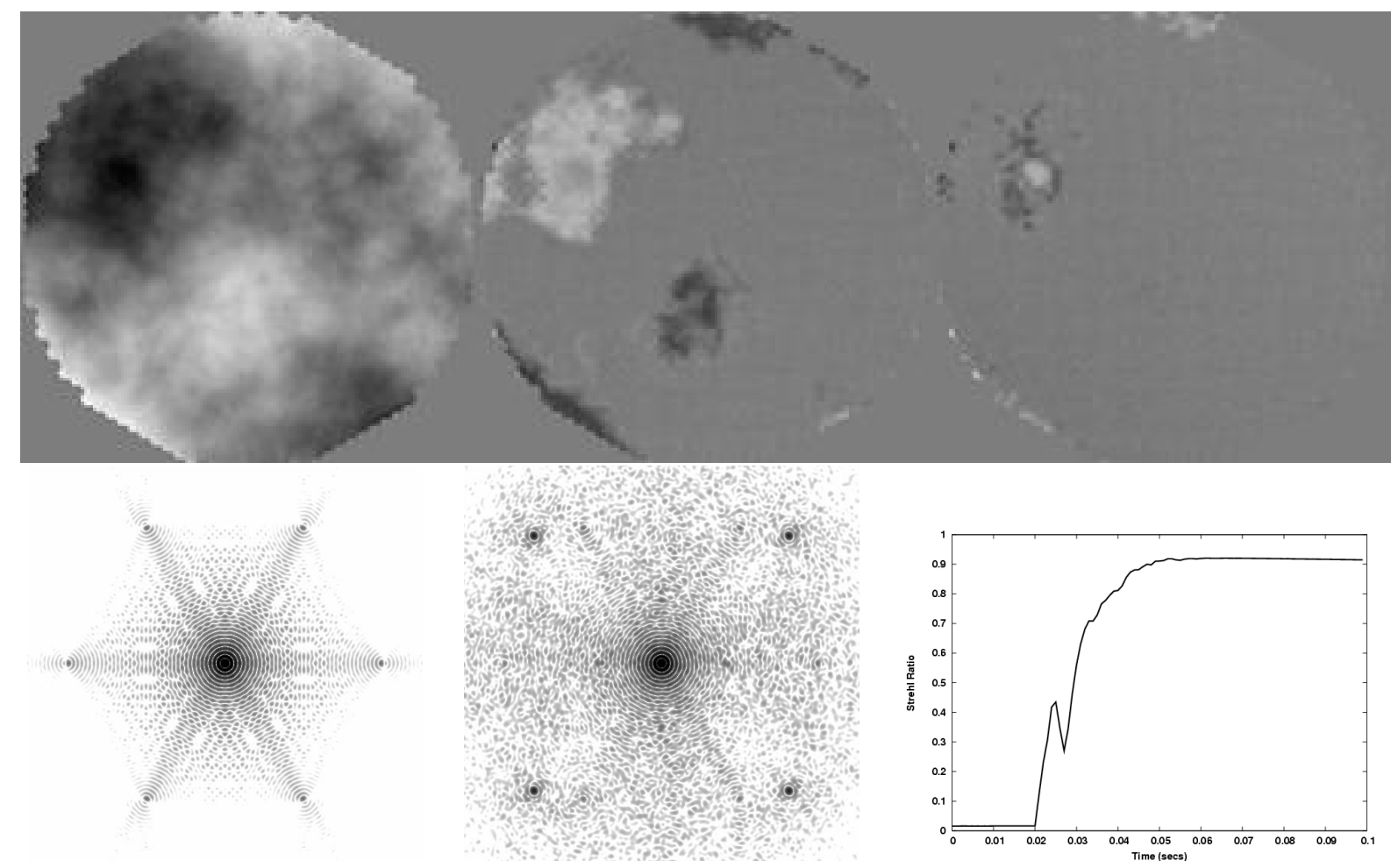

Figure 2. Closed loop adaptive optics simulation on a 30 meter segmented mirror telescope with a $64 x 64$ subaperture AO system. The upper set of images shows the residual wavefront error in the pupil plane at three different timesteps in the simulation. The first shows the residual phase errors after the tip tilt loop has stabilized, while the second and third show the residual phase errors after 10 and 20 cycles of the high order control loop, respectively. The lower set of images shows the the PSF in the absence of atmospheric turbulence, the AO compensated PSF, and the Strehl ratio history. The PSF's are displayed on a log stretch clipped at $10^{-3}$ of full scale.

Also shown in Figure 2 are the PSF in the absence of turbulence and the turbulence-corrupted PSF after compensation by the adaptive optics system. These PSF's both display sixfold symmetry arising from diffraction off of the hexagonal segments. The AO compensated PSF also displays a fourfold symmetry arising from waffle on the deformable mirror. Figure 2 shows the Strehl ratio history over the course of the simulation, which displays the interesting feature that the Strehl ratio does not monotonically increase with time. This effect arises because different patches in the pupil interfered destructively.

\section{ACTUATOR TO SUBAPERTURE REGISTRATION}

The most commonly used registration between the actuators and subapertures of a single conjugate adaptive optics system with a Shack Hartmann sensor is the Fried geometry. In this geometry, the actuators are located at the corners of the subapertures. An alternative is the Southwell geometry, in which the actuators are centered on the subapertures. More generally, the actuators and subapertures may be registered in arbitrary geometries, which may be classified using six degrees of freedom. Two of these correspond to relative lateral displacements of actuators and subapertures. An overall rotation and a magnification of the actuator grid with respect to the subapertures constitute two more degrees of freedom. Finally, the actuator grid may be tilted with respect to the subapertures, so that the grid appears foreshortened along one axis. Examples of three different geometries resulting from varying the lateral displacement of the actuators and subapertures appear in Figure 3. Alternative geometries might only be of academic interest were it not for the fact that realistic optical systems must introduce misregistration at some level. Reconstructors generated for the particular geometry afforded by the optical system may then provide an improvement over the use of a reconstructor that assumes no misregistration. An AO system 


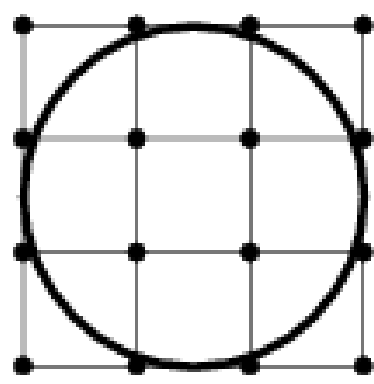

Fried

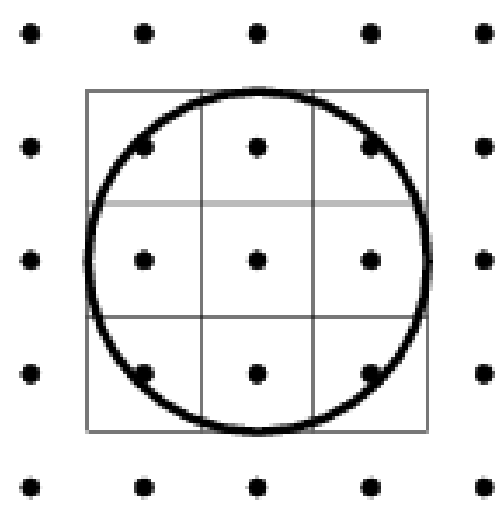

Southwell

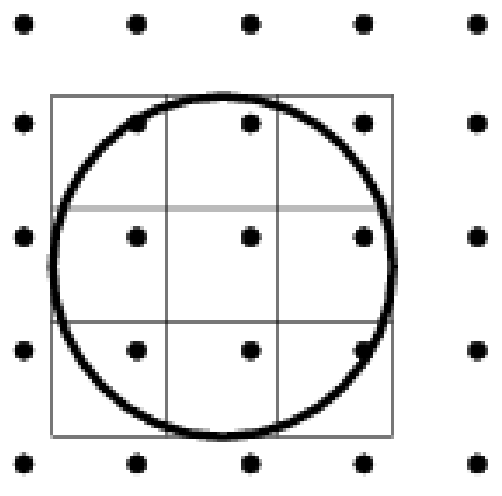

Offset

Figure 3. Three examples of the geometrical registrations of actuators to subapertures. In these figures, dots represent actuators, the square grid represents the subapertures, and the circle represents the aperture of the telescope. On the left is pictured the Fried geometry, in which actuators are placed at the corners of the subapertures. The central image shows the Southwell geometry, in which actuators are centered on the subapertures. On the right, a geometry with an arbitrary offset between actuators and subapertures is shown.

designer faces the choice of establishing a tolerance on the optical alignment of the AO system such that the performance degradation is negligable, or of choosing to use a reconstructor tuned to the known misregistration.

This section presents two different studies related to these effects. The first investigates the closed loop performance of adaptive optics systems for lateral displacements of the actuator and subaperture grids when the reconstructors are generated for the true geometrical configuration. The second study investigates the performance degradation of an adaptive optics system under lateral displacements of the actuator and subaperture grids about the Fried geometry, when the reconstructor does not account for these perturbations.

In the first study, the actuator grid was offset relative to the subaperture grid along a 45 degree diagonal, starting from the Fried geometry and ending at the Southwell geometry. A reconstructor suitable for use with a 32x32 subaperture adaptive optics system was generated for each geometrical configuration. A closed loop simulation was then performed for a 5 meter aperture, with wind velocities of the turbulent layers set to zero. The results of this study are shown in Figure 4, and show a degreadation in system performance as one moves away from the Fried geometry. Physically, this may be understood by considering the centroid value generated by displacing a single actuator at the center of a subaperture in the Southwell geometry. The measured centroid is given by the integral of the phase gradient over the subaperture, which cancels out identically for this geometry. This cancellation leads to a suppression of the eigenvalue spectrum and a corresponding degradation in system performance. In contrast, there is no cancellation in the Fried geometry. For intermediate geometries the cancellation is incomplete, leading to intermediate levels of performance.

The second study aimed to investigate the stability of an AO system in the presence of misregistration when using a reconstructor generated for the Fried geometry. In this study, a fixed subaperture size of $36 \mathrm{~cm}$ was chosen, and the aperture diameter of the telescope was varied between 2.5 and 12.5 meters. This corresponded to AO systems of order $7 \times 7$ to $35 \times 35$. For each configuration, a series of simulations was performed in which the deformable mirror was shifted with respect to the lenslet array by increasing amounts. At some offset, the stability of the high order loop began to degrade. An illustration of this behavior is shown in Figure 5 . The offset at which the loop began to destabilize is indicative of the tolerance of the AO system to misregistration. This tolerance is plotted as a function of aperture diameter in Figure 5. These results are loosely consistent with the observed tolerance to misregistration in the Palomar AO system, which is thought to be of order .15 subapertures. ${ }^{5}$ While one can expect that this tolerance will generally depend on seeing and wind conditions, the results indicate that much tighter tolerances to misregistration are required for large actuator count $\mathrm{AO}$ 

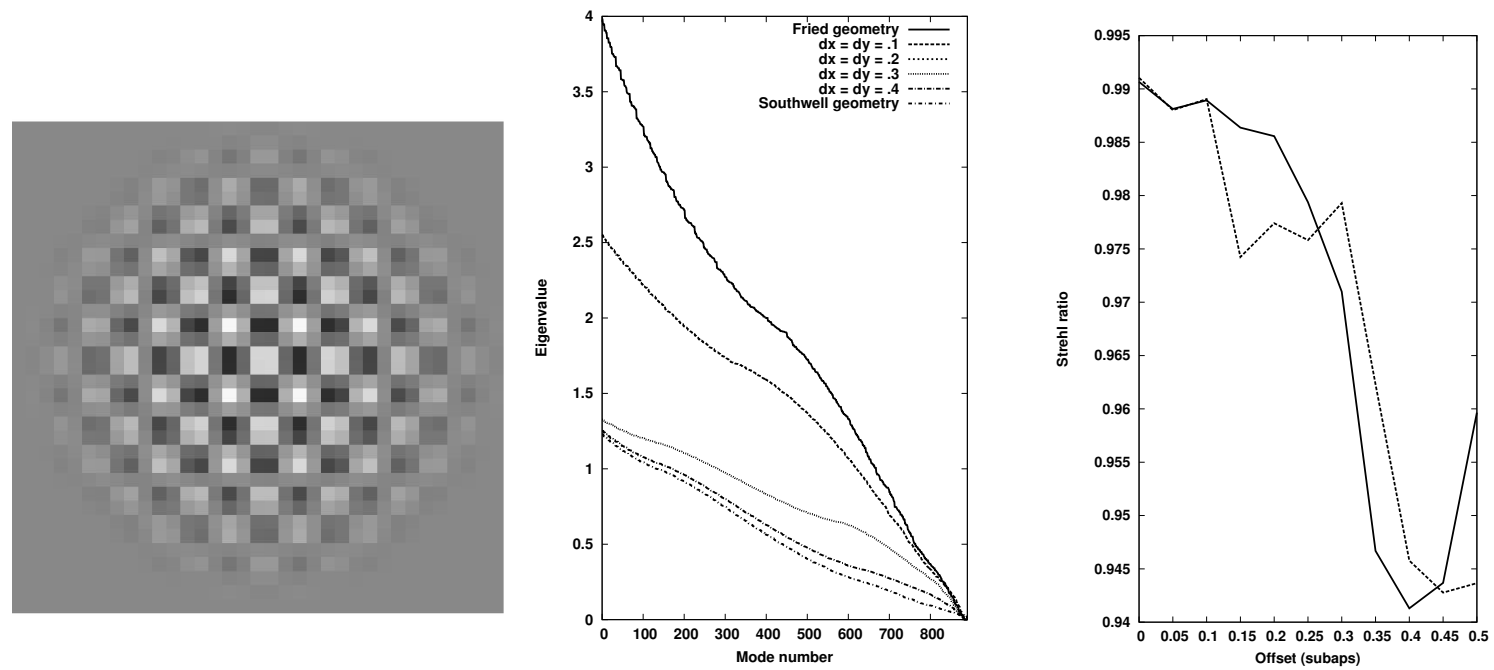

Figure 4. Reconstruction using geometries with lateral displacements of the actuator and subaperture grids. On the left is shown the mode with the largest eigenvalue for a 32x32 subaperture system in the Southwell geometry. The center plot shows the eigenvalue spectrum for reconstructors generated for geometries offset along a 45 degree diagonal. Zero offset corresponds to the Fried geometry, while an offset of half a subaperture in the $\mathrm{x}$ and y lateral displacement corresponds to the Southwell geometry. The right plot shows the Strehl ratio as a function of reconstructor geometry for two random realizations of atmospheric turbulence.

systems on large apertures. This sensitivity could be a significant problem for the high order AO systems now being planned for large aperture telescopes.

The signature for the onset of the high order loop instability is increased levels of waffle in the deformable mirror, with an accompanying increase in amplitude of the waffle spots in the AO compensated PSF. Physically, this effect arises because an offset in the registration of the deformable mirror and the lenslet array changes the eigenvector structure of the geometry matrix. Because one is misinterpreting the eigenvectors of the offset geometry as those of the Fried geometry when applying corrections to the deformable mirror, introduction of a Fried eigenmode onto the wavefront results in many modes being placed onto the deformable mirror. And because the singular modes evolve with the geometric registration, misregistration can place unsensed modes onto the deformable mirror that are sensed in the geometry assumed by the reconstructor. For nearly Fried geometries, these modes look very much like waffle. Over many cycles of the control loop these modes can build up on the deformable mirror, leading to the performance degradation seen in Figure 5. This is also a signature of other types of misregistration, such as rotation and magnification.

\section{TIP TILT PROJECTION IN ACTUATOR AND CENTROID SPACE}

Single conjugate adaptive optics systems that rely on a deformable mirror to perform adaptive correction typically perform tip tilt correction with a separate actively controlled flat mirror. This factorization of the correction is motivated by the fact that most of the wavefront error resides in tip and tilt. By compensating these modes with a separate mirror, the stroke requirements on the deformable mirror may be considerably reduced. The reconstructor typically performs this factorization by generating separate estimates for the tip tilt and high order residuals from the centroid measurements. The tip tilt estimate may be made in one of two ways: by averaging the centroid measurements to obtain an estimate, or by reconstructing the actuator commands and then projecting out the tilt. The former technique provides an estimate of the gradient tilt, while the latter provides an estimate of the Zernike tilt. ${ }^{6}$ The Zernike tilt corresponds to the correction provided by the tip tilt 

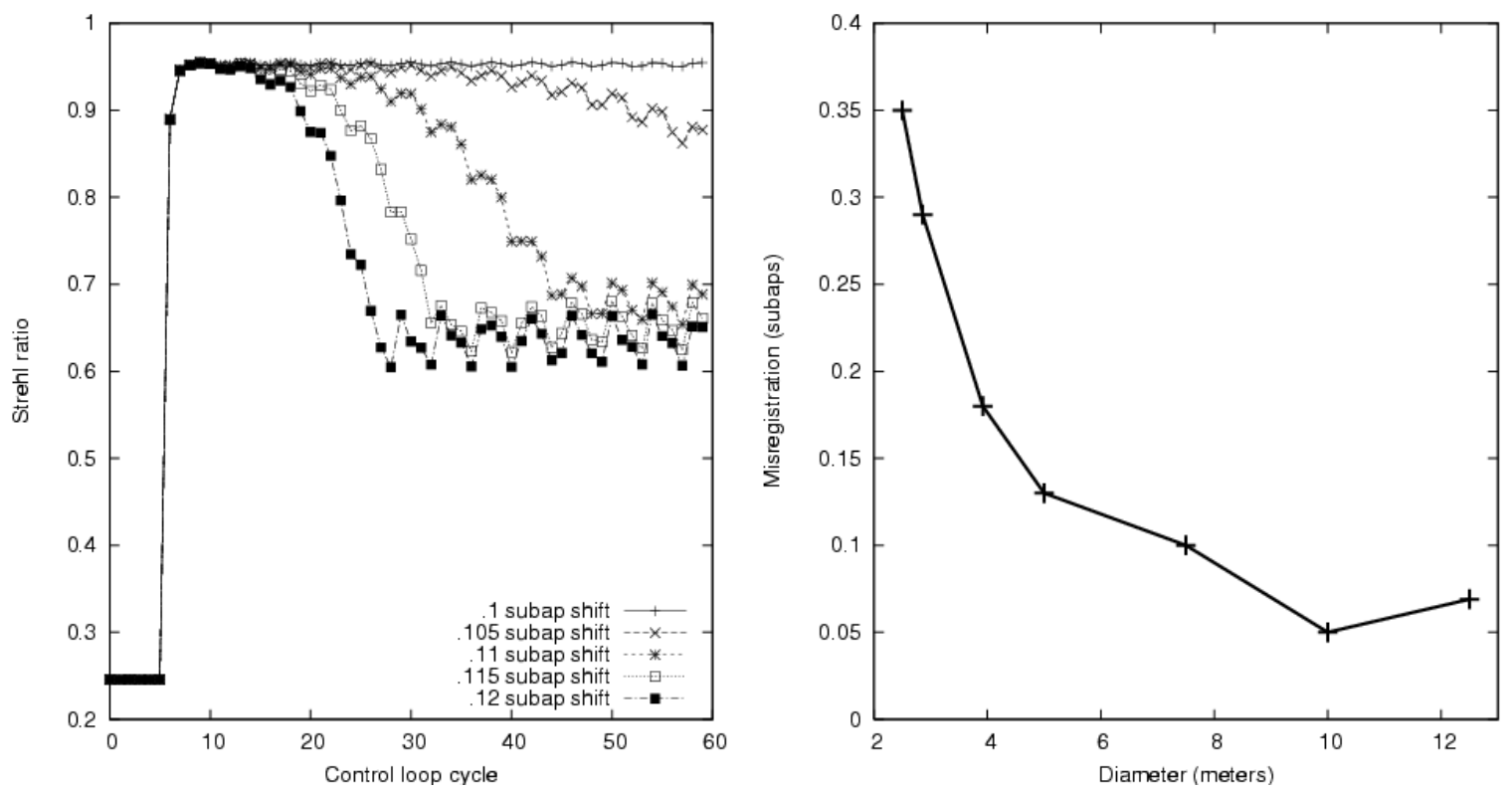

Figure 5. Loop stability in the presence of actuator to lenslet misregistration. The left figure shows the temporal evolution of the Strehl ratio for different levels of deformable mirror to lenslet array misregistration, for a telescope with a 7.5 meter aperture and an AO system with 21 subapertures across the pupil. The onset of loop instability occurs at a misregistration of .105 subapertures. On the right is plotted the misregistration tolerance as a function of aperture diameter. These simulations were performed with a fixed subaperture size of $36 \mathrm{~cm}$, and the number of subapertures across the pupil varied from 7 to 35 for apertures between 2.5 and 12.5 meters in diameter.

mirror, and use of the gradient tilt to drive this mirror results in a small amount of Zernike tilt being placed on the deformable mirror. While these estimates differ only by a few percent, the differences may accumulate over many cycles of the control loop to generate a large effect. The final study presented in this paper investigates the control loop dynamics for reconstructors that perform tip tilt projection in actuator space and in centroid space.

The simulations employed an annular aperture with inner and outer diameters of 1.89 and 5.08 meters, which correspond to the values of the Hale telescope at Palomar. An AO system with 16x16 subapertures was used in this study. Wind velocities of the atmospheric phase screens were randomly drawn from a model of the vertical wind profile, ${ }^{1}$ and had magnitudes from $5 \mathrm{~m} / \mathrm{s}$ at the ground up to $25 \mathrm{~m} / \mathrm{s}$ at $15 \mathrm{~km}$. The simulation was executed on 1 millisecond timesteps, and the control loops were updated at this rate. To facilitate a direct comparison between the two reconstruction schemes, the simulations were repeated for each reconstruction technique using the same set of atmospheric phase screens.

The amplitude of the tip tilt in the uncorrected wavefront phase and on the tip tilt and deformable mirrors are shown as a function of time in Figure 6. The reconstruction algorithm that performs tip tilt projection in centroid space has a tendency to place tilt onto the deformable mirror and compensate this using the tip tilt mirror. This is particularly evident at the beginning and towards the end of the simulation. In contrast, the reconstruction algorithm that performed tip tilt projection in actuator space placed less tilt onto the deformable mirror. It should be emphasized that employing tip tilt projection in centroid space does not lead to a degradation in system performance unless the deformable mirror runs out of stroke.

\section{CONCLUSIONS}

This paper has presented a series of numerical simulations intended to explore some properties of single conjugate adaptive optics systems. Studies of high order control loop dynamics on large aperture telescopes and 

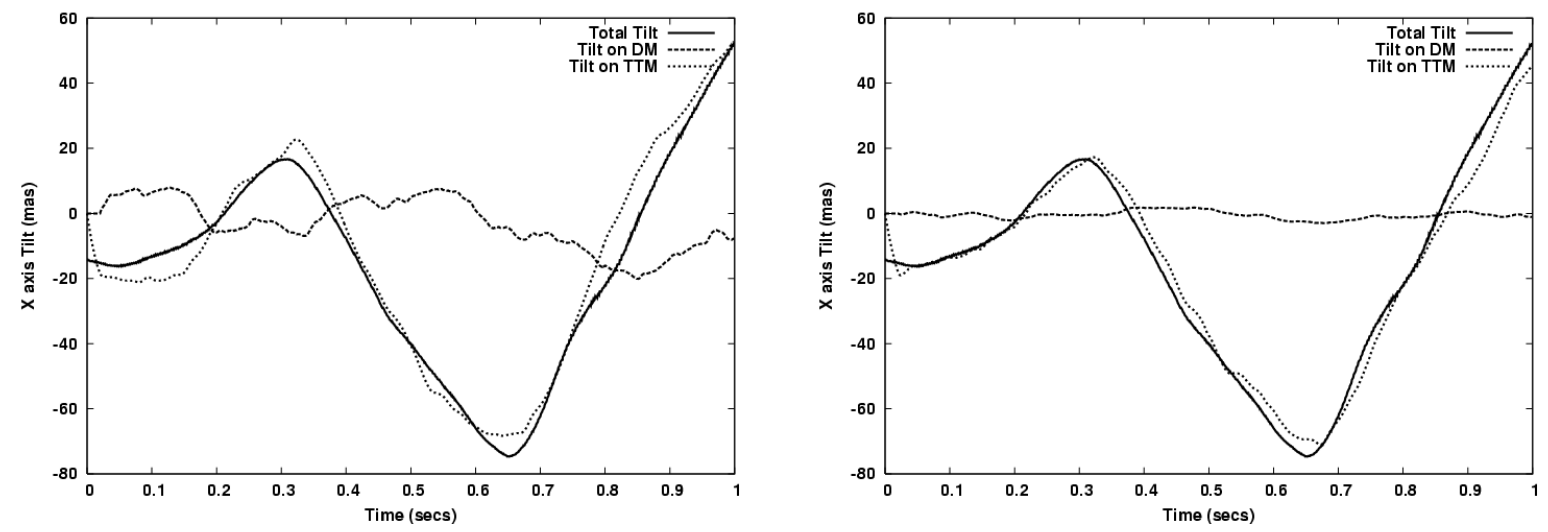

Figure 6. Time histories of the $\mathrm{X}$ tilt component on the uncompensated wavefront phase, and on the tip tilt and deformable mirrors. The left plot shows the $\mathrm{X}$ tilt history when the reconstructor performs tip tilt projection in centroid space. Because of the slight difference between Zernike and gradient tilt, tilt can build up on the deformable mirror over time. This can be a problem for mirrors with finite stroke. The right plot shows the tilt history for a reconstructor that performs tip tilt projection in actuator space. The tilt component on the deformable mirror is suppressed by this reconstructor.

of misregistration tolerance as a function of the order of the AO system both suggest potential difficulties in constructing the next generation of high actuator count AO systems on large aperture telescopes. Quantitative predictions of reconstructor performance like those presented here may be carried out in numerical simulation, and may serve to test reconstructor proposals under repeatable conditions. These types of studies are difficult to carry out in analysis, and are illustrative of the regime in which time domain numerical simulations are uniquely suited to answer questions in the design and analysis of adaptive optics systems.

\section{ACKNOWLEDGEMENTS}

The author would like to thank Richard Dekany for many useful discussions, and Doug MacMynowski for suggesting the study presented in Section 5 and providing the reconstructors.

The Thirty Meter Telescope (TMT) Project is a partnership of the Association of Universities for Research in Astronomy (AURA), the Association of Canadian Universities for Research in Astronomy (ACURA), the California Institute of Technology and the University of California. The partners gratefully acknowledge the support of the Gordon and Betty Moore Foundation, the US National Science Foundation, the National Research Council of Canada, the Natural Sciences and Engineering Research Council of Canada, and the Gemini Partnership.

\section{REFERENCES}

1. J. W. Hardy, Adaptive Optics for Astronomical Telescopes, Oxford University Press, New York, 1998.

2. M. C. Britton, "Arroyo," these proceedings , 2004.

3. R. G. Lane, A. Glindemann, and J. C. Dainty, "Simulation of a komolgorov phase screen," Waves in Random Media 2, p. 209, 1992.

4. B. L. Ellerbroek and F. J. Rigaut, "Scaling multiconjugate adaptive optics performance estimates to extremely large telescopes," in Proc. SPIE Vol. 4007, p. 1088-1099, Adaptive Optical Systems Technology, Peter L. Wizinowich; Ed., pp. 1088-1099, July 2000.

5. R. G. Dekany. personal communication, 2004.

6. R. J. Sasiela, Electromagnetic Wave Propagation in Turbulence, Springer-Verlag, New York, 1994. 